\title{
Analysis of Two Methods of Poles Extraction for Antenna Caracterization
}

\author{
F. Sarrazin, A. Sharaiha \\ IETR, University of Rennes 1, UEB \\ Campus de Beaulieu, Rennes, France \\ francois.sarrazin@univ-rennes1.fr
}

\author{
P. Pouliguen, P. Potier, J. Chauveau \\ Direction Générale de l'Armement (DGA) \\ France \\ philippe.pouliguen@dga.defense.gouv.fr
}

\begin{abstract}
Antenna radiation characteristics can be modeled by some poles and residues. In this paper, we compare two different methods to extract these poles of a noisy antenna radiation in frequency and in time domain: the Cauchy method and the Total Least Square Matrix Pencil Method respectively. Results are presented for a dipole antenna and an Archimedean spiral antenna.
\end{abstract}

\section{INTRODUCTION}

Since many years, the Singularity Expansion Method (SEM) [1], introduced by C. E. Baum in 1971, is studied to be applied to target identification. In fact, SEM represents a solution of an electromagnetic problem in terms of singularities (poles). Since singularities are independent of the direction of the incoming wave, it makes it useful for scatter identification. More recently, this method has been applied in both time and frequency domains to model antenna effective length, in order to fully describe the antenna pattern, directivity and gain using only a few set of parameters (poles and residues) [2] [3]. They are extracted using different methods. In frequency domain, we can use the Cauchy method. In time domain, the most popular one is the Matrix Pencil Method (MPM). The purpose of this paper is to compare these methods applied on antenna radiation responses and to analyze their robustness to Signal-to-Noise Ratio (SNR), which to the author's knowledge has never been done. In section II, we recall both methods. In section III, we compare these methods applied on dipole and Archimedean spiral antenna responses in presence of noise.

\section{SEM THEORY}

\section{A. Total Least Square Matrix Pencil Method (TLS-MPM)}

In time domain, Hua and Sarkar [4] propose to model the noisy data set $y_{k}$ as

$$
y_{k} \approx \sum_{n=1}^{N} R_{n} e^{s_{n} k}=\sum_{n=1}^{N} R_{n} z_{n}^{k}
$$

where $N$ is the number of poles, $k=0,1, \ldots, K-1, K$ is the number of sampled data $(K>2 N), R_{n}$ the residues, $s_{n}$ the poles $\left(s_{n}=\sigma_{n} \pm j \omega_{n}\right.$ with $\sigma_{n}$ the damping coefficient and $\omega_{n}$ the resonant pulsation). A data matrix $[Y]$ is built (2) in compliance with the pencil parameter $L$, useful in reducing noise in the data and generally chosen between $K / 3$ and $K / 2$. Then, a Singular Value Decomposition is carried out as $[Y]=[U][\Sigma][V]^{H}, H$ being the Hermitian transpose, $[U]$ and $[V]$ are unitary matrices composed of the eigenvectors of
$[Y][Y]^{H}$ and $[Y]^{H}[Y]$, respectively. $[\Sigma]$ is a diagonal matrix containing the singular values of $[Y]$.

$$
[Y]=\left[\begin{array}{cccc}
y_{0} & y_{1} & \ldots & y_{L} \\
y_{1} & y_{2} & \ldots & y_{L+1} \\
\vdots & \vdots & \ddots & \vdots \\
y_{K-L-1} & y_{K-L} & \ldots & y_{K-1}
\end{array}\right]
$$

A significant parameter $M$ is now chosen such that the singular values in $\left[\sum\right]$ beyond $M$ are "small" and can be approximated by zero. A reduced matrix $\left[V^{\prime}\right]$ can now be constructed using only the rows corresponding to the $M$ most significant value as $\left[V^{\prime}\right]=\left[\begin{array}{llll}v_{1} & v_{2} & \cdots & v_{M}\end{array}\right]^{T}$. Next, we define two submatrices $\left[\mathrm{V}_{1}{ }^{\prime}\right]$ and $\left[\mathrm{V}_{2}{ }^{\prime}\right]$ from [ $\left.\mathrm{V}^{\prime}\right]$ by deleting the last column of [V'] and the first column of [V'], respectively. The problem is reduced to a left-hand eigenvalue problem given by (3). The eigenvalues $\lambda$ correspond to poles of the system in terms of $z^{k}$.

$$
\left[V_{2}^{\prime}\right]=\lambda\left[V_{1}^{\prime}\right] \rightarrow\left[V_{2}^{\prime}\right]\left[V_{1}^{\prime}\right]^{H}=\lambda\left[V_{1}^{\prime}\right]\left[V_{1}^{\prime}\right]^{H}
$$

\section{B. Cauchy Method}

Another method is the Cauchy method [5]. It is applied on a transfer function in frequency domain. The transfer function $\mathrm{H}(\mathrm{s})$ is approximated by a ratio of two polynomials as

$$
H(s)=\frac{P_{M}(s)}{Q_{N}(s)}=\frac{\sum_{n=0}^{M} a_{m} s^{m}}{1+\sum_{n=1}^{N} b_{n} s^{n}}
$$

where $N=M+1$ is the filter order. Equation (4) can also be written as a matrix equation $A x=b$ with $x=\left[\begin{array}{llllll}b_{1} & \cdots & b_{N} & -a_{0} & \cdots & -a_{M}\end{array}\right]^{T}$ which is solved using a least square approach as $x=\left(A^{H} A\right)^{-1} A^{H} b$. The transfer function $H(s)$ can now be modeled as

$$
H(s)=\sum_{n=1}^{N} \frac{R_{n}}{s-s_{n}} \square
$$

\section{APPLICATION ON NOISY ANTENNA RESPONSES}

\section{A. Dipole Antenna}

To compare the robustness of the methods, we first consider a dipole antenna. Its length is $\mathrm{L}=33.75 \mathrm{~mm}$, its radius is $R=0.56 \mathrm{~mm}$ and its gap is $\mathrm{G}=0.56 \mathrm{~mm}$. It is simulated in frequency domain using HFSS [6] between 1 and $18 \mathrm{GHz}$ with 
$\delta \mathrm{f}=17 \mathrm{MHz}$. The noiseless E-farfield radiated by the dipole antenna in the boresight direction is presented Fig. 1. Cauchy is now applied on this response. An Inverse Fast Fourier Transform (IFFT) is also performed and MPM is applied on the transient response obtained. Poles extracted using both methods are presented Fig. 2 . We note that poles are very close and they correspond to the two resonances of the dipole radiation (at $\lambda / 2$ and $3 \lambda / 2$ ). Only the damping coefficient of the second pole (at $12.2 \mathrm{GHz}$ ) differs from only $0.210^{9} \mathrm{rad} / \mathrm{s}$. Next, we compare these methods in presence of Gaussian white noise added to obtain different Signal to Noise Ratio (SNR) from -10 to 70 $\mathrm{dB}$. For each SNR, ten different sets of noisy data have been used. The number of poles extracted by each method is presented in Table I. A pole is considered well extracted when the resonant frequency is the same than without noise within $5 \%$. When SNR $=10 \mathrm{~dB}$, MPM still extracts the four poles of the response while Cauchy extracts only two poles.

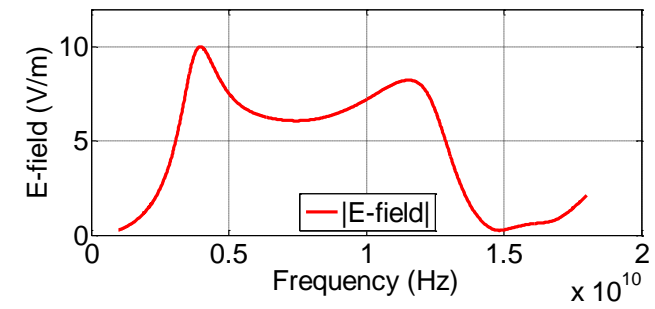

Figure 1. Noiseless E-field of the dipole antenna.

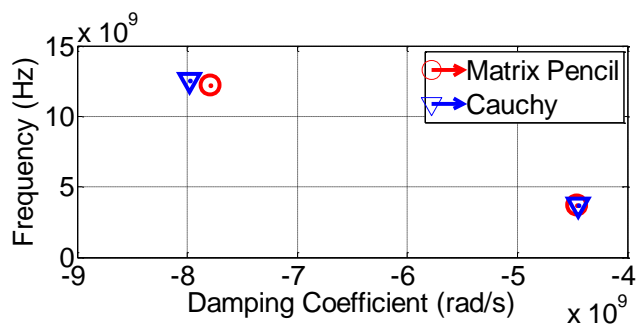

Figure 2. Poles extracted without noise.

TABLE I. NUMBER OF POLES EXTRACTEd VERSUS SNR.

\begin{tabular}{|c|c|c|c|c|c|c|c|c|c|}
\hline \multirow{2}{*}{ Method } & \multicolumn{10}{|c|}{ SNR (dB) } \\
\cline { 2 - 11 } & $\mathbf{- 1 0}$ & $\mathbf{0}$ & $\mathbf{1 0}$ & $\mathbf{2 0}$ & $\mathbf{3 0}$ & $\mathbf{4 0}$ & $\mathbf{5 0}$ & $\mathbf{6 0}$ & $\mathbf{7 0}$ \\
\hline Cauchy & 0 & 0 & 2 & 4 & 4 & 4 & 4 & 4 & 4 \\
\hline MPM & 0 & 2 & 4 & 4 & 4 & 4 & 4 & 4 & 4 \\
\hline
\end{tabular}

\section{B. Archimedean Spiral Antenna}

We now consider a one and a half tour UWB Archimedean spiral antenna which radius is $13 \mathrm{~mm}$ and is matched between 4.5 and $18 \mathrm{GHz}$. It is simulated using HFSS between 1 and 18 $\mathrm{GHz}$ with $\delta \mathrm{f}=17 \mathrm{MHz}$. Fig. 3 presents the noiseless E-farfield in the boresight direction. As for the dipole, Cauchy is applied on this response and MPM is applied on its IFFT. Results are presented in Fig. 4. We observe that the first pole (at $3.4 \mathrm{GHz}$ ) is well extracted by both methods. For the other poles, resonant frequencies are almost the same but damping coefficients differ. We add a Gaussian white noise to the response and apply both methods. Results are presented in Table 2. When SNR becomes low, MPM still extracts some poles while Cauchy doesn't.

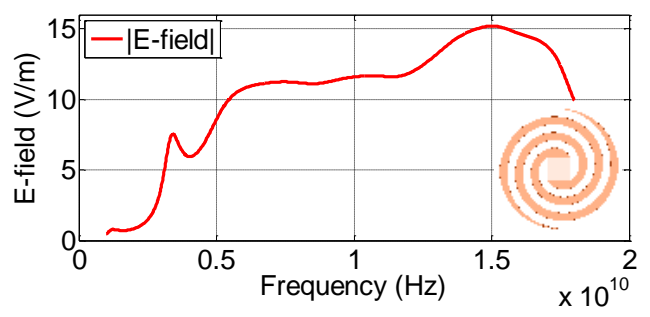

Figure 3. Noiseless E-field of the Archimedeam antenna.

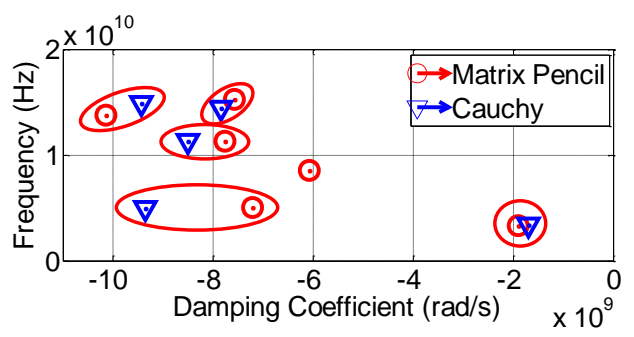

Figure 4. Poles extracted without noise.

TABLE 2. Number OF POLES EXTRACTED VERSUS SNR.

\begin{tabular}{|c|c|c|c|c|c|c|c|c|c|}
\hline \multirow{2}{*}{ Method } & \multicolumn{10}{|c|}{ SNR (dB) } \\
\cline { 2 - 11 } & $\mathbf{- 1 0}$ & $\mathbf{0}$ & $\mathbf{1 0}$ & $\mathbf{2 0}$ & $\mathbf{3 0}$ & $\mathbf{4 0}$ & $\mathbf{5 0}$ & $\mathbf{6 0}$ & $\mathbf{7 0}$ \\
\hline Cauchy & 0 & 2 & 4 & 6 & 6 & 6 & 6 & 8 & 10 \\
\hline MPM & 2 & 4 & 4 & 4 & 6 & 6 & 8 & 12 & 12 \\
\hline
\end{tabular}

IV. CONClusion AND PERsPeCtives

To our knowledge, these results are the first comparison between MPM and Cauchy method to analyze antenna radiated responses. In both cases, MPM extracts more poles than Cauchy method when SNR becomes low. Moreover, according to the response, the order of the filter must be modified to help the Cauchy algorithm to converge. MPM doesn't need any modification and is more computational. Additional analyses are in progress to confirm these first interesting results, for example by filtering the early time of radiation responses.

\section{ACKNOWLEDGEMENT}

This work was financially supported by the DGA.

\section{REFERENCES}

[1] C. E. Baum, "On the singularity expansion method for the solution of electromagnetic interaction problems", EMP Interaction Note 8, Air Force Weapons Laboratory, Kirkland AFB, New Mexico, Dec. 1971.

[2] S. Licul and W. A. Davis, "Unified frequency and time-domain antenna modeling and characterization", IEEE Transactions on Antenna and Propagation, vol. 53, No 9, pp. 2882-2888, Sep. 2005.

[3] C. Marchais, B. Uguen, A. Sharaiha, G. L. Ray and L. Le Coq, "Compact characterisation of ultra wideband antenna responses from frequency measurements", IET, Microwaves, Antennas \& Propagation, vol. 5 , Issue: 6, pp. 671-675, 2011.

[4] Y. Hua and T. K. Sarkar, "Matrix pencil method for estimating parameters of exponentially damped/undamped sinusoids in noise", IEEE transactions on Acoustics, speech and signal processing, vol. 38, No , pp. 814-824, May. 1990.

[5] A. L. Cauchy, "Sur la formule de Lagrange relative à l'interpolation", Analyse Algèbrique, Paris, 1821.

[6] Ansys HFSS, Available: http://www.ansoft.com/products/hf/hfss/. 